archives-ouvertes

\title{
Fault Identification on a Fuel Cell by 3-D Current Density Reconstruction From External Magnetic Field Measurements
}

Lyes Ifrek, Olivier Chadebec, Sebastien Rosini, Gilles Cauffet, Yann Bultel, Bertrand Bannwarth

\section{To cite this version:}

Lyes Ifrek, Olivier Chadebec, Sebastien Rosini, Gilles Cauffet, Yann Bultel, et al.. Fault Identification on a Fuel Cell by 3-D Current Density Reconstruction From External Magnetic Field Measurements. IEEE Transactions on Magnetics, Institute of Electrical and Electronics Engineers, 2019, 55 (6), pp.6200705. 10.1109/TMAG.2019.2895755 . hal-02350903

\section{HAL Id: hal-02350903 \\ https://hal.archives-ouvertes.fr/hal-02350903}

Submitted on 14 Nov 2019

HAL is a multi-disciplinary open access archive for the deposit and dissemination of scientific research documents, whether they are published or not. The documents may come from teaching and research institutions in France or abroad, or from public or private research centers.
L'archive ouverte pluridisciplinaire HAL, est destinée au dépôt et à la diffusion de documents scientifiques de niveau recherche, publiés ou non, émanant des établissements d'enseignement et de recherche français ou étrangers, des laboratoires publics ou privés. 


\title{
Fault Identification on a Fuel Cell by 3D Current Density Reconstruction from External Magnetic Field Measurements
}

\author{
Lyes Ifrek ${ }^{1,2}$, Olivier Chadebec ${ }^{1}$, Sébastien Rosini ${ }^{3}$, Gilles Cauffet $^{1}$, Yann Bultel ${ }^{2}$, Bertrand Bannwarth ${ }^{1}$ \\ ${ }^{1}$ Univ. Grenoble Alpes, CNRS, Grenoble INP, G2Elab, F-38000 Grenoble, France \\ ${ }^{2}$ Univ. Grenoble Alpes, CNRS, Grenoble INP, LEPMI, F-38000 Grenoble, France \\ ${ }^{3}$ Univ. Grenoble Alpes, CEA, LITEN, F-38054 Grenoble, France
}

\begin{abstract}
An original approach of fuel cell diagnosis is presented. It is based on the solving of an inverse linear problem linking the magne tic field signature outside of the fuel cell to the current density distribution inside. The searched solution is a linear combination of conservative current distribution obtained by a set of electrokinetic problems solved by a finite face element method. As the problem is ill-posed, the solution is stabilized using a truncated singular value decomposition. The approach is validated to reconstruct a 3D current density distribution in a stack simulator and in a fuel cell stack operating in laboratory conditions.
\end{abstract}

Index Terms - Fuel cell, non-invasive diagnosis, current density identification, magnetic measurements, inverse problem.

\section{INTRODUCTION}

$\mathrm{F}$ UEL cell stack is an electrochemical device which produces electrical energy from a reaction of hydrogen and oxygen and is obtained by assembling elementary cells in series.Up to now, cost and durability of fuel cells represent the most significant challenges to achieve clean, reliable, and cost-effective systems, in particular for transport applications. Multiphysic phenomena in a fuel cell expose the different materials of the stack (membrane, electrodes, bipolar plate, etc.) to several constraints on some parts of the stack. Repeated constraints lead inevitably to the degradation of fuel cell components. According to the material heterogeneity or simply operating conditions within the stack, the current density distribution became heterogeneous. The knowledge of local current density flowing within the system is fundamental to prevent components ageing. The total current produced by the stack is easy to measure by using an ammeter but getting the local one needs the use of some more sophisticated methods. As an example, an invasive $\mathrm{S}^{++}{ }^{\circledR}$ device can be used to direct measure the local current distribution in some part of the stack section but this invasive system presents the drawback to disturb the stack operation.

To avoid interferences with the stack operation, noninvasive methods based on the external magnetic field measurements has been proposed [1]-[2]. This original technique uses magnetic sensors placed around the stack to measure the magnetic signature of the current distribution inside it. In [1], the magnetic field cartography around a stack is obtained by using a 3-axis magnetic sensor mounted on a moving robotic arm. One hundred points of measurement were needed to get a good resolution for the current cartography. The important drawback of such a tool is the time of the acquisition and the calculation which is about 15 minutes. In such a time, the internal state of the stack can be modified, so the current density estimation can be wrong. A more recent work is based on the solving of an optimization problem [3]. It proposes to minimize an objective function defined as the difference between the real magnetic measurements and those obtained by random combinations of current densities. The magnetic field is obtained from 2000 configurations realized on 36 magnetic three-axis sensors. The model which is computationally expensive has been validated on a device containing only one cell but not on a whole stack.

On our previous works [4]-[5], an alternative approach has been proposed. It is based on an original reconstruction of a current density basis by using Fourier series coupled to a finite volume modelling of a stack. A sensors array configuration which is only sensitive to the current's inhomogeneity due to faults occurring inside the stack has also been proposed. It was demonstrated that a set of only 30 external and fixed magnetic measurements around a stack could be used to build a 2D current density distribution in a short time (i.e $1 \mathrm{~min}$ ). However, the approach has been developed only for the diagnosis of global faults which affects identically all the cells of the stack. This is why the denomination of 2D fault identification has been adopted. In this paper, we go a step forward by adapted this approach to more localised faults involving only few adjacent cells in a stack and by proposing an original 3D faults identification method.

\section{3D MAGNETIC TOMOGRAPHY APPROACH}

\section{A. Forward modelling}

Changes of the operating conditions (stoichiometry, relative humidity) and apparition of local faults (hot spots, materials degradation) modify the current density distribution inside a fuel cell stack [4]. In this section, a model is proposed to simulate the current and streamline distributions taking into account these effects by considering a local change of the conductivity in the stack.

The forward modelling is divided in two steps. The first step consists in computing the current density knowing the electric conductivity and the global current flowing into the stack (solving of an electrokinetic problem). The second step is to compute the magnetic field outside (i.e in the air region) from the current density distribution (solving of a magnetos tatic problem).

The electrokinetic problem is solved by the Finite Element Method (FEM). The geometry is discretized and the conservative current flux vector quantity $\mathbf{j}$ is interpolated by face shape functions (Whitney 2-form). This choice of shape 
function is justified by the fact that they naturally ensure the conservation of the flux of current density between two adjacent elements. Two equations have to be solved simultaneously:

$$
\begin{gathered}
\operatorname{div}(\mathbf{j})=0 \\
\frac{\mathbf{j}}{\sigma}=-\operatorname{grad} V
\end{gathered}
$$

where $V$ is the electric potential and $\sigma$ the conductivity. A Galerkin's projection of the first equation on face shape functions $\boldsymbol{\omega}$ leads to a linear matrix system:

$$
[\mathbf{R}] \cdot \mathbf{I}=\mathbf{U}
$$

where

$$
\begin{aligned}
& \mathbf{R}_{i j}=\int_{\Omega s} \frac{\boldsymbol{\omega}_{i} \cdot \boldsymbol{\omega}_{j}}{\sigma} d \Omega_{s} \\
& \mathbf{U}_{i}=-\int_{\Omega s} \boldsymbol{\omega}_{i} \cdot \operatorname{grad} V d \Omega_{s}
\end{aligned}
$$

and $\mathbf{I}$ is a vector containing the flux flowing in the faces of the mesh. It has been demonstrated in [6] that (3) can be interpreted as the matrix representation of an equivalent electric circuit composed in this case of a network of resistances. In this circuit, each face of the mesh is associated to a resistance and each element of the mesh to a circuit node.

It remains to ensure the current solenoidality of $\mathbf{j}$ i.e the equation (1). It can be achieved by finding a set of equivalent independent loops in the circuit in order to change the problem:

$$
[\mathbf{M}]^{\mathrm{T}} \cdot[\mathbf{R}] \cdot[\mathbf{M}] \cdot \mathbf{I}_{\text {loop }}=[\mathbf{M}]^{\mathrm{T}} \cdot \mathbf{U}=\mathbf{U}_{\mathbf{S}}
$$

where $[\mathbf{M}]$ is the branch-fundamental independent loop incidence matrix (where the value of each element can be $-1,0$ or 1), $\mathbf{U}_{\mathbf{s}}$ is the vector of source voltages (often equal to 0 ) and Iloop the current flowing into independent loops. The matrix system is solved by a linear solver and face currents are obtained by applying the reverse transform process:

$$
\mathbf{I}=[\mathbf{M}]^{\mathrm{T}} \cdot \mathbf{I}_{\text {loop }}
$$

This formulation ensures the free-divergence of the current which is fundamental in order to get an accurate computation of the external magnetic field like in [4]. Moreover, this approach can deal with any discretized element-type and is easy and natural to couple with external electric circuit. It is why FEM has been preferred to finite volume method initially proposed in [4].

Once FEM electrokinetic problem has been solved and current distribution density has been computed, a magnetostatic problem has to be solved in order to compute the magnetic field at any point around the stack. It can be efficiently achieved by numerically integrating the Biot-Savart law because of the absence of ferromagnetic material:

$$
\mathbf{B}(\mathbf{r})=\frac{\mu_{0}}{4 \pi} \int_{\Omega_{s}} \mathbf{j}\left(\mathbf{r}_{s}\right) \times \frac{\mathbf{r}-\mathbf{r}_{s}}{\left|\mathbf{r}-\mathbf{r}_{s}\right|^{3}} d \Omega_{s}
$$

where $\mathbf{B}$ is the magnetic induction at position $\mathbf{r}, \mathbf{j}$ the current density at the integrating point $\mathbf{r}_{\mathrm{s}}, \Omega_{\mathrm{s}}$ the stack domain and $\mu_{0}$ the magnetic permeability of the vacuum.

\section{B. Inverse problemparametrization}

\section{1) Generalities}

The problem being linear, the current density in the case of a faulty operation mode is the sum of two modes: A common mode, which corresponds to a reference state of the stack, and a differential mode, which corresponds to the variation of the current density caused by a fault (Fig.1.).

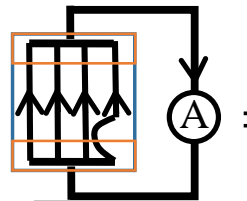

(a)

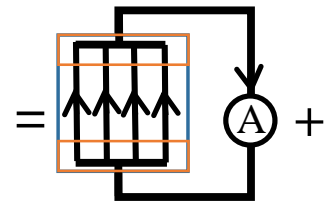

(b)

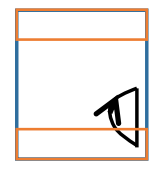

(c)
Fig. 1. Current mode decomposition [4]. Total curren t mode (a); co mmon current mode (b) and differential current mode (c).

Both modes create an external field defined as the sum of:

$$
\mathbf{B}_{\text {tot }}=\mathbf{B}_{c}+\Delta \mathbf{B}_{d}
$$

where $\mathbf{B}_{t o t}$ is the magnetic induction created by the total mode, $\mathbf{B}_{c}$ the magnetic induction created by the common mode and $\Delta \mathbf{B}_{d}$ the magnetic induction created by the differential mode. We assume that the reference state is known because it corresponds to the healthy state (or a reference one) and it can be determined by an ammeter which measures the total current flowing into the stack. $\mathbf{B}_{c}$ can then be determined by modelling or better by measurement on the reference state. By subtracting it to the measurements, $\Delta \mathbf{B}_{d}$ can be estimated. The Biot-Savart law being linear, the numerical integration of (8) leads to the following matrix system:

$$
\Delta \mathbf{B}_{d}=[\mathbf{S}] \cdot \Delta \mathbf{j}_{d}
$$

where $\mathbf{S}$ is the discretized Biot-Savart operator and $\Delta \mathbf{j}_{d}$ and $\Delta \mathbf{B}_{d}$ define the discretized differential current and differential magnetic field respectively. It remains to find adequate basis for both currents and magnetic measurements to efficiently parameterise the inverse problem.

\section{2) Choice of the current density basis}

In a first step, the FEM problem is solved to determine the common mode. An average electrical conductivity of $5 \times 10^{3} \mathrm{~S}$ $\mathrm{m}^{-1}$ is chosen for the active region [5]. The conductivity for end plates is $5 \times 10^{7} \mathrm{~S} \mathrm{~m}^{-1}$, classically much higher than the previous one. A reference value for current density $\mathbf{j}_{c}$ is computed considering a healthy stack. In order to compute $\Delta \mathbf{j}_{d i}$ the element $i$ of $\Delta \mathbf{j}_{d}$, the stack domain is divided into $5 \times 5 \times 5$ volume parts. A zero electric conductivity is set to one of the 125 volumes, all the other ones being set to the normal conductivity. The problem is solved thanks to the FEM electrokinetic model and the current density obtained is subtracted to $\mathbf{j}_{c}$ in order to get a $\Delta \mathbf{j}_{d i}$ current distribution. $\Delta \mathbf{B}_{d}$ is computed using the Biot-Savart law (8). The process is repeated 125 times, setting successively a null conductivity to each volume in order to get the whole basis. Another current basis has been investigated in [7] but according to our experience, this current basis seems more efficient.

\section{3) Choice of the magnetic field basis}

In previous works [4], the sensors axes are oriented in order to measure only the differential magnetic field (radial 
direction) and to be insensitive to the common mode. In this previous study, only one sensors array of 30 magnetic sensors was used and was located in the middle of the stack (middle sensor array Fig. 2). In this study and in order to get more information about the stack state along its axis, the sensor array has been translated (Fig. 2) and the magnetic field has been measured on three different planes (middle of the stack, middle left and middle right). Two components are measured, the radial $\left(\boldsymbol{B}_{\mathrm{r}}\right)$ one and the axial one $\left(\boldsymbol{B}_{\mathrm{a}}\right)$.

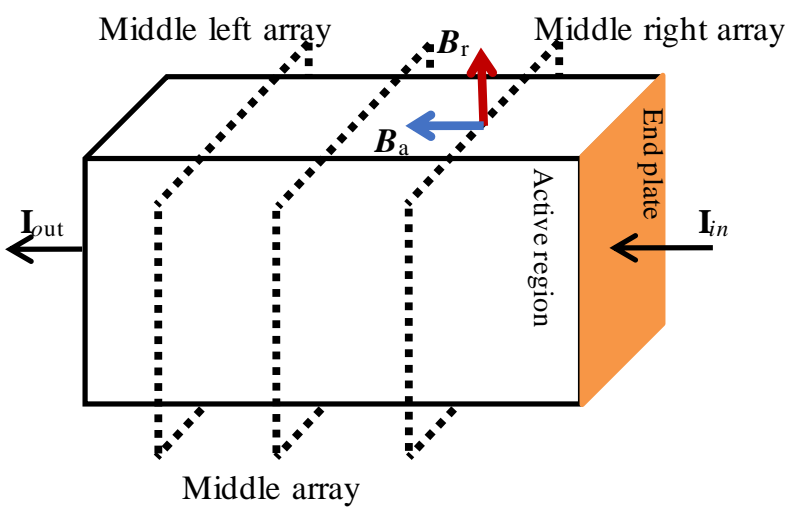

Fig. 2: Sensors arrays locations around a fuel cell stack

Two examples of faults among 125 are presented in Fig. 3a. The corresponding magnetic signatures to both previous current density vectors are also presented (Fig. 3b).

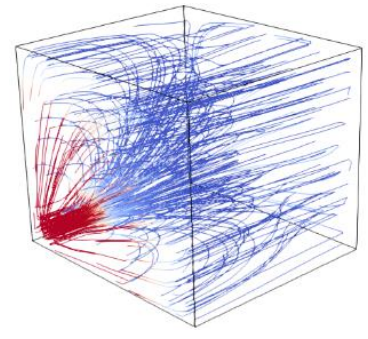

$\Delta \mathbf{j}_{d_{1}}$

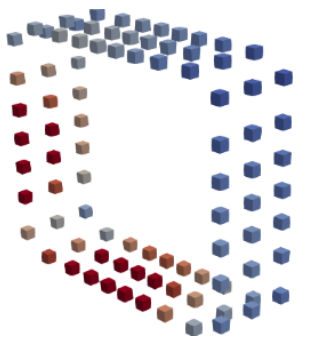

$\Delta \mathbf{B}_{d_{1}}$

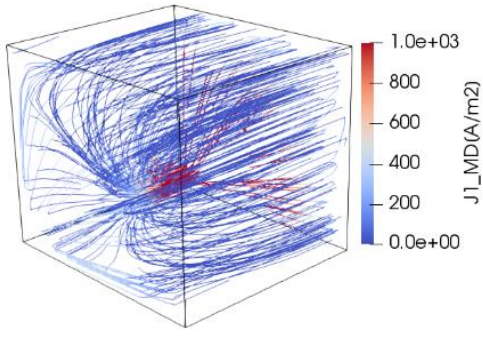

$\Delta \mathbf{j}_{d_{2}}$

(a)

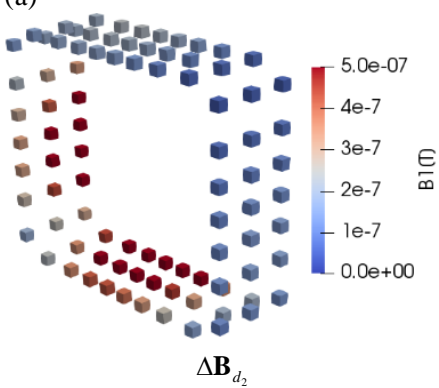

(b)

Fig. 3: Example of two vectors used to build the current and magnetic basis: Current vectors (a) and related magnetic field magnitude vectors (b)

\section{4) Inverse problem resolution}

The inverse problem is ill-posed meaning that the solution is not unique and very sensitive to the magnetic noise. In order to regularize the solution, a classical truncated pseudo-inverse approach is used [8]. A Singular Value Decomposition (SVD) of $[\mathbf{S}]$ is made and its spectrum is truncated to get $\left[\mathbf{S}_{\text {trunc }}\right]$ where small singular values which amplify the measurement noise have been suppressed. The solution is obtained with:

$$
\Delta \mathbf{j}_{d}=\left[\mathbf{S}_{\text {trunc }}\right]^{\#} \Delta \mathbf{B}_{d}
$$

where $\left[\mathbf{S}_{\text {trunc }}\right]^{\#}$ is the pseudo inverse of $\left[\mathbf{S}_{\text {trunc }}\right]$.

As the approach is validated with a stack simulator and a Proton Exchange Membrane Fuel Cell stack with different sections and length, the number of sensors is different for each device (30 and 24). The system of equations is overdetermined and contains 180 (real stack) or 144 (stack simulator) equations (number of sensors $\mathrm{x} 3$ positions $\mathrm{x} 2$ components of the magnetic field) for 125 unknowns.

\section{EXPERIMENTALRESULTS}

The developed approach has first been tested on a stack simulator in order to validate the methodology. Finally, the systemhas been deployed around a real fuel cell stack.

\section{A. Stack simulator results}

Two graphite blocks with a cut part (Fig. 4a and Fig. 4b) has been built in order to mimic a faulty stack with an important conductivity change (cuts of $24 \%$ and $3 \%$ of the volume respectively). The common mode is determined by measuring the field around the same graphite block but without the cut part. Each graphite block is caught between copper end plates and supplied by an external circuit. The stack simulator including the external magnetic field measurements system of 24 sensors is presented on Fig. 4. The sensors array is translated along the stack simulator length to perform measurements on the three locations (see Fig. 2). In order to maximize the signal to noise ratio, sensors are located as close as possible to the stack simulator $(35 \mathrm{~mm})$.

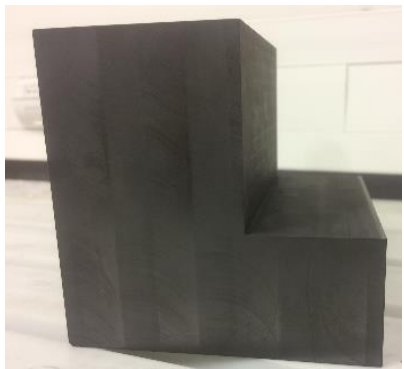

(a)

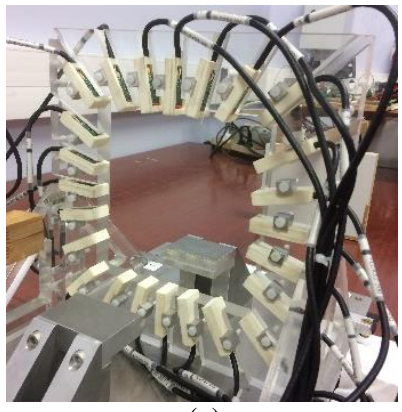

(c)

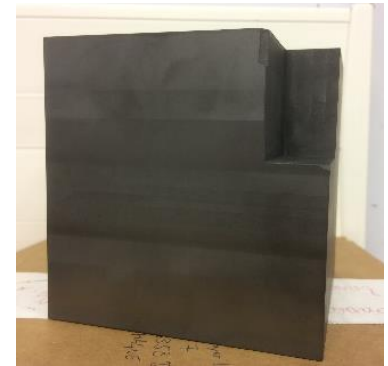

(b)

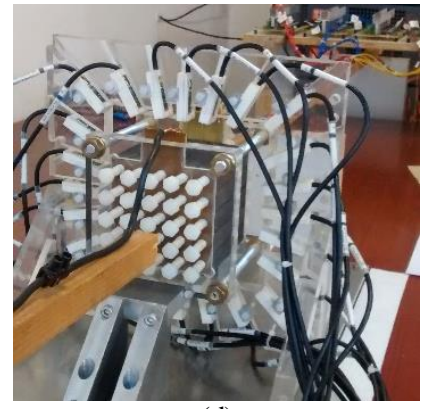

(d)
Fig.4: Stack simulator experiment: Graphite block with 24\% cut part (a), Graphite block with $3 \%$ cut part (b); Sensors array (c) and whole device wit h sensors array $(\mathrm{d})$

The magnetic field is recorded at $\mathrm{I}=25 \mathrm{~A}$ and the common mode measurement is subtracted to get only the differential 
one. The inverse problem is solved and the current density distribution is obtained for both configurations (Fig. 5b and Fig. 5d). The difference $\varepsilon$ between the simulated current density distributions target (Fig. 5a and Fig. 5c) and the identified one is calculated with (12) and it is about $25 \%$ and $35 \%$.

$$
\varepsilon=\sqrt{\frac{\int_{\Omega_{s}}\left(\mathbf{j}_{t a r}-\mathbf{j}_{i d n}\right)^{\mathrm{T}} \cdot\left(\mathbf{j}_{t a r}-\mathbf{j}_{\text {idn }}\right) d \Omega_{s}}{\int_{\Omega_{s}} \mathbf{j}_{\text {tar } \max }^{\mathrm{T}} \cdot \mathbf{j}_{\text {tar } \max } d \Omega_{s}}}
$$

where $\mathbf{j}_{t a r}$ is the simulated current density distribution, $\mathbf{j}_{\text {idn }}$ the identified current density distribution and $\mathbf{j}_{\text {tarmax }}$ is the maximal current target value.

In the both cases, the current reconstruction shows a low current density close to the faults location.

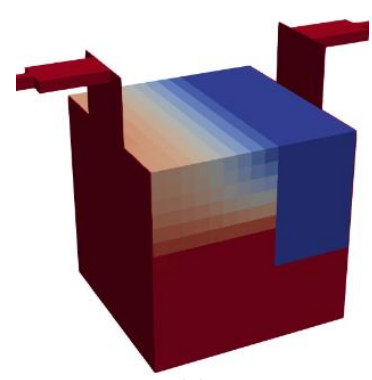

(a)

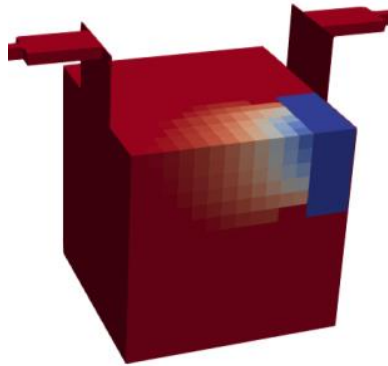

(c)

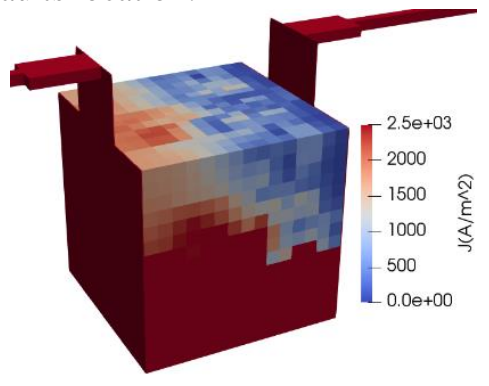

(b)

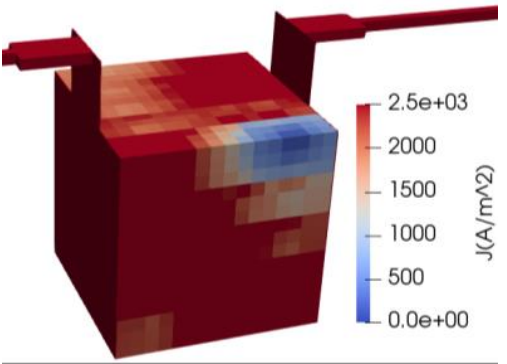

(d)
Fig.5: Result s obtained with the stack simulator. Simulated current den sity $24 \%$ cut part (a); identified one from external magnetic field $24 \%$ cut part (b); Simulated current density $3 \%$ cut part (c) and identified on e from ex ternal magnetic field $3 \%$ cut part (d).

\section{B. Fuel cell stack result}

A GENEPAC fuel cell stack with 100 cells [5] (Fig. 6.a) is used to validate methodology. To do this, five cells, shown on Fig. 6b, are voluntary damaged and as sembled on the stack.

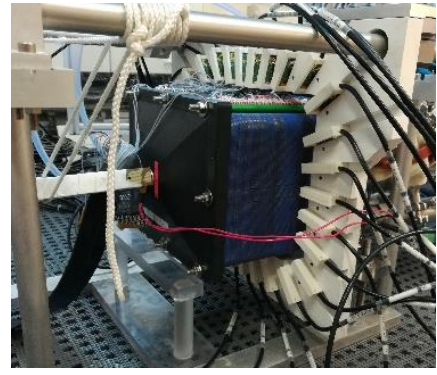

(a)

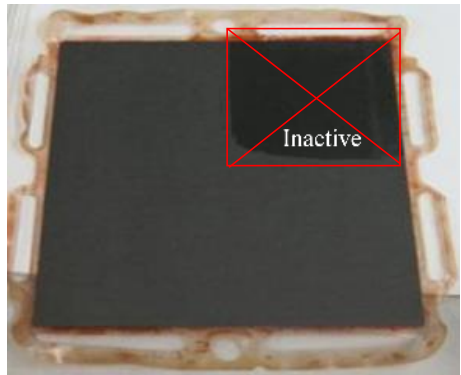

(b)
Fig.6: Real stack experiment: GENEPAC Fuel Cell stack surrounded by a magnetic sensors array (a); Example of MEA with $20 \%$ of the active area inhibited using a resin (b).

The identified differential current mode is shown in Fig. 7.
As expected, the identified current density distribution becomes heterogeneous in the plane containing the fault and it is highly modified to force the current to pass through the healthy part of the cells because of the higher resistance caused by an inactive area (Fig. 6b). The measurements process is made in less than 1 minute and the computation time is very low $(<1 \mathrm{~s})$, the $125 \mathrm{FEM}$ problems being solved only one time before inverse resolution.

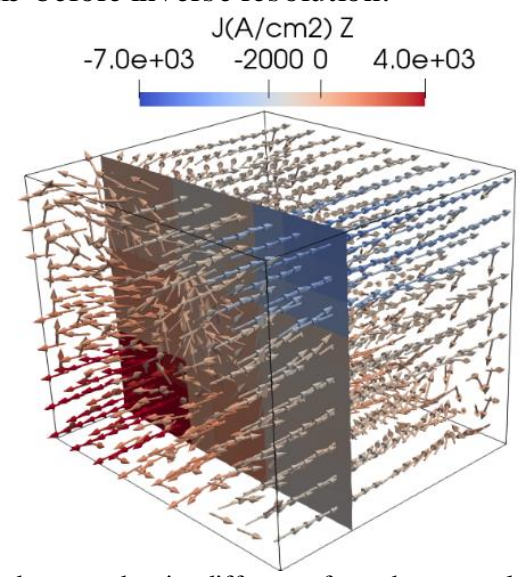

Fig.7: Identified current density difference from the external magnetic field. The magnetic measurements have been made at $\mathrm{I}=100 \mathrm{~A}$ for reference conditions to get the common mode.

\section{CONCLUSION}

A magnetic tomography approach to locate different kinds of faults on a fuel cell was presented. The tool makes possible the identification of the 3D current distributions in the fuel cell independently of the size of the fault within the limit of the precision of the sensors. The validation has been carried out on both a simulator and a real fuel cell stack.

\section{ACKNOWLEDGMENT}

This work was funded by the Auvergne-Rhône-Alpes region.

\section{REFERENCES}

[1] K.H. Hauer, R. Potthast, T. Wüster and D. Stolten, "Magnetotomography: a new method for analysing fuel cell performance and quality," J Power Sources, vol. 143, p. 67-74, 2005.

[2] H. Lustfeld, M. Reißel, and B. Steffen "Magnetotomography and electric currents in a fuel cell." Fuel Cells, vol. 4, p. 474-481,2009.

[3] R. Yamanashi, Y. Gotoh, M. Izumi, and T. Nara, "Evaluation of Generation Current inside Membrane Electrode Assembly in Polymer Electrolyte Fuel Cell Using Static Magnetic Field around Fuel Cell," ECS Trans., vol. 65, no 1, 2015.

[4] M. Le Ny, O. Chadebec, G. Cauffet, J. M. Dedulle, Y. Bultel, S. Rosini, Y. Fourneron and P. Kuo-Peng, "Current Distribution Identification in Fuel Cell Stacks From External Magnetic Field Measurements," IE EE Trans. Magn., vol. 49, no 5, p. 1925-1928, 2013.

[5] M. Le Ny, O. Chadebec, G. Cauffet, R. Rosini, and Y. Bultel, "PEMFC stack diagnosis based on external magnetic field, measurements," J Appl Electrochem., vol.45, p 667-677, 2015.

[6] T.T. Nguyen, G. Meunier, J. M Guichon, O. Chadebec, and T. S. Nguyen. "An integral formulation for the computation of 3-D eddy current using facet elements," IEEE Trans. Magn., vol. 50, no. 2, p. 549$552,2014$.

[7] L. Ifrek, O. Chadebec, G. Cauffet, Y. Bultel, S. Rosini, L. Rouveyre, "Spatial harmonic current density basis for faults identification in fuel cell stack from external magnetic field measurements'" International Applied Computational, 2017.

[8] P. Xu, "T runcated SVD methods for discrete linear ill-posed problems," Geophys. J. Int.vol.135, p.505-514, 1998. 